International Journal of Applied Engineering Research

ISSN 0973-4562 Volume 10, Number 9 (2015) pp. 21777-21783

(C) Research India Publications

https://dx.doi.org/10.37622/IJAER/10.9.2015.21777-21783

\title{
Reviewing Otsu's Method For Image Thresholding
}

\author{
Sunil L. Bangare \\ Research Scholar, Dept. of Computer Science \& Engineering, \\ KL University, Green Fields, Vaddeswaram, \\ Guntur District-522 502, A.P., India
}

Amruta Dubal

Dept. of Information Technology,

Sinhgad Academy of Engineering,

Kondhwa-Bk, Pune-48, Maharashtra, India

Pallavi S. Bangare

Assistant Professor, Dept. of Information Technology,

Sinhgad Academy of Engineering, Kondhwa-Bk, Pune-48, Maharashtra, India

\author{
Dr. S. T. Patil \\ Professor, Dept. of Computer Engineering, \\ Vishwakarma Institute of Technology, \\ Pune-37, Maharashtra, India.
}

\begin{abstract}
Image processing is largely used for gathering more knowledge / understanding either by human or by machines like computer. Segmentation, Thresholding and Edge detection are an important technique in Computer vision and Image processing. In digital images feature detection or extraction can be done for finding the irregularities in the image maybe in the rightness etc. This paper is a small review on Otsu's method. This is proposed for improving the efficiency of computation for the optimal thresholds of an image.

This paper gives thresholding technique and Otsu's method of thresholding, also expresses its algorithm and working. This method gives satisfactory results when the numbers of pixels in each class are close to each other. It is the most referenced thresholding methods, as it directly operates on the gray level histogram, so it's fast and computes an optimized threshold value. It automatically performs clustering-based image thresholding as its one of many binarization algorithms.
\end{abstract}


Index Terms: Grayscale, Histogram, Otsu, Pixel, Thresholding.

\section{Introduction}

Thresholding is method used to remove an intended object or target object from its background image by allocating an value of intensity $\mathrm{T}$ (threshold) for every pixel such as each pixel is either categorized as an background point or a object point. In various applications of image processing, the gray levels of pixels belonging to the object materially vary from the gray levels of the pixels belonging to the background.

Thresholding then becomes a simple but effective tool to separate objects from the background. Examples of thresholding applications are document image analysis, where the goal is to extract printed characters, logos, graphical content, or musical scores: map processing, where lines, legends, and characters are to be found: scene processing, where a target is to be detected: and quality inspection of materials, where defective parts must be delineated. Other applications can be listed as follows: cell images and knowledge representation [2],

Otsu's thresholding method implicates iterating through all the credible threshold values and enumerating a measure of spread for the pixel levels each side of the threshold, i.e. the pixels that are in foreground or background. The intent is to decide the threshold value where the summation of foreground and background escalates is at its minimum.
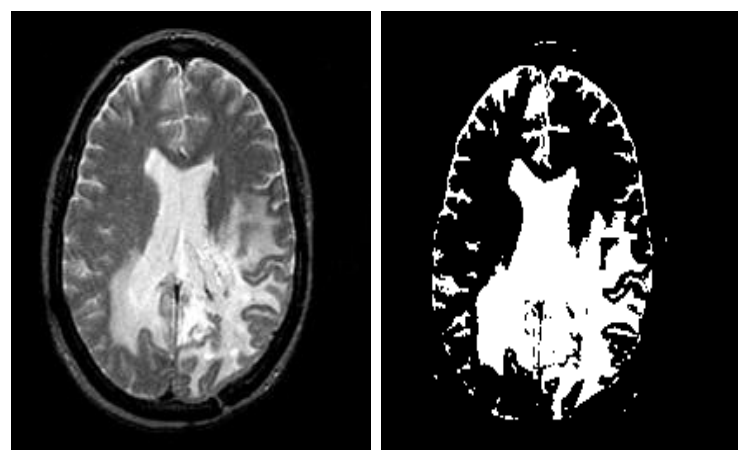

Figure 1: Thresholding of brain tumor from grayscale MRI image

Generally intensity is the easiest property which is shared by 'pixels' in particular region. So thresholding is normally used for the segmenting light and dark regions. Here grey level image is converted in to binary image by changing all of the pixels below certain threshold value to ZERO and all above threshold value to ONE.

The simplest such abstraction is the process of image thresholding, which can be thought of as an extreme form of gray-level quantization. Suppose that a gray-level image $f$ can take $\mathrm{K}$ possible gray levels $0,1,2, \ldots, \mathrm{K}-1$. Define an integer threshold, $\mathrm{T}$, that lies in the gray-scale range of $\mathrm{T}$ lies between $(0,1,2, \ldots, \mathrm{K}-1)$. The process of thresholding is a process of simple comparison: each pixel value in $f$ is compared to threshold, T. Based on this comparison; a binary decision is made that defines the value of the corresponding pixel in an output binary image $g$. 
If $g(x, y)$ is a thresholded version of $f(x, y)$ at some global threshold $T$,

$$
g(x, y)= \begin{cases}1 & \text { if } f(x, y) \geq T \\ 0 & \text { otherwise }\end{cases}
$$

\section{Algorithm:}

Step 1: Compute histogram for a 2D image.

Step 2: Calculate foreground and background variances (measure of spread) for a single threshold.

i) Calculate weight of background pixels and foreground pixels.

ii) Calculate mean of background pixels and foreground pixels.

iii)Calculate variance of background pixels and foreground pixels.

Step 3: Calculate "within class variance"

\section{Logic}

\section{Assumptions:}

- To simplify the explanation Consider only 6 grayscale levels are used.

- Consider threshold value 3 for 6grayscale levels.

1) Calculate weight for background and foreground pixels

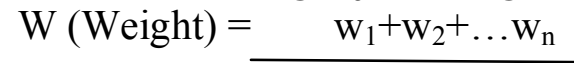

Total number of pixels

\section{1) Calculate mean}

Histogram value $=h$

$\mu($ Mean $)=\left(\mathrm{h}_{1} * \mathrm{w}_{1}\right)+\left(\mathrm{h}_{2} * \mathrm{w}_{2}\right)+\ldots\left(\mathrm{h}_{\mathrm{n}} * \mathrm{w}_{\mathrm{n}}\right)+\left(\mathrm{h}_{\mathrm{n}} * \mathrm{w}_{\mathrm{n}}\right)$

Sum of weights (W)

\section{2) Calculate variance}

$$
\mathrm{V}=\left(\left(\mathrm{h}_{1}-\mu\right)^{2 *} \mathrm{w}_{1}\right)+\left(\left(\mathrm{h}_{2}-\mu\right)^{2 *} \mathrm{~W}_{2}\right) \ldots\left(\left(\mathrm{h}_{\mathrm{n}}-\mu\right)^{2}{ }^{2} \mathrm{w}_{\mathrm{n}}\right)+\left(\left(\mathrm{h}_{\mathrm{n}}-\mu\right)^{2}{ }^{2} \mathrm{w}_{\mathrm{n}}\right)
$$

Sum of weights (W)

\section{3) Calculate within class variance}

I:e sum of two variances multiplied by their associated weights.

Within class variance $=\mathrm{W}_{\mathrm{b}} * \mathrm{~V}_{\mathrm{b}}+\mathrm{W}_{\mathrm{f}} * \mathrm{~V}_{\mathrm{f}}$

\section{Drawbacks of Thresholding}

The main problem with this method is the dependency on 'intensity' as no other 
relationship of pixels is considered. Also pixels at the boundary region and sometimes the shadows of the object create problem.

Also noise will affect the thresholding. Illumination in the scene may have some lower or higher brighter parts so global thresholding will not be useful and local thresholding is preferred. Automated thresholding methods can be used for finding thresholds.

\section{Otsu's Thresholding Method}

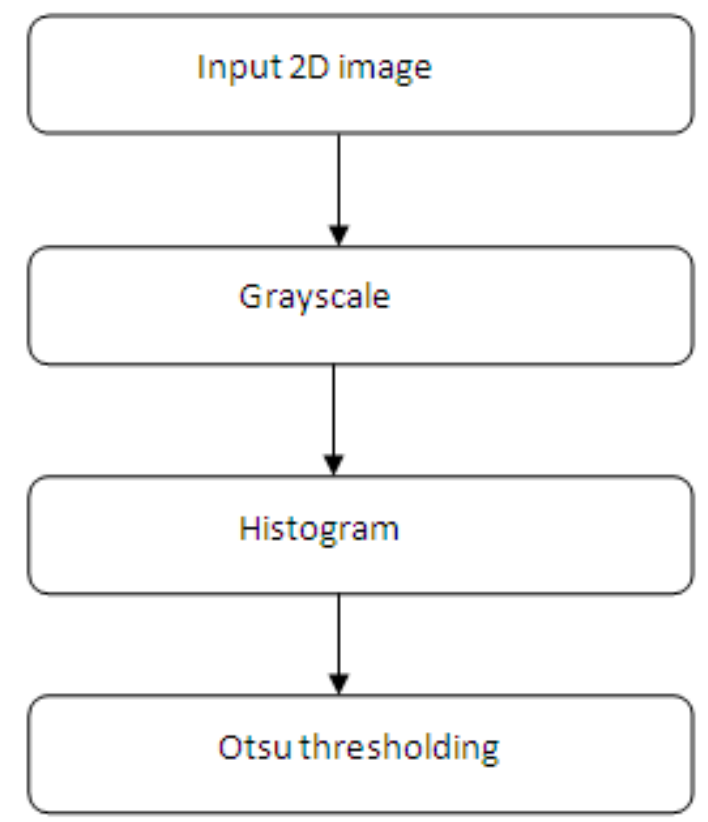

Figure 2: Flow of Otsu's thresholding in Image Processing

In optimal thresholding, a "criterion function" is formulated that yields some measure of dissociation between regions. A criterion function is computed for intensity and that which maximizes this function is selected as the threshold. Otsu's thresholding picks the threshold value to minimize the intra-class variance of the thresholded black and white pixels. A specific criterion function is used as a measure of statistical separation for descriptive analysis. First, a criterion for maximizing a modified between-class variance that is equivalent to the criterion of maximizing the usual between-class variance is proposed for image segmentation. Next, in accordance with the new criterion, a recursive algorithm is designed to efficiently find the optimal threshold. Converting a grayscale image to monochrome is a common image processing task.

Otsu's method instinctively performs clustering-based image thresholding, or, the diminishing of a grayscale image to a binary or threshold image. The algorithm considers that the image contains two classes of pixels backing bi-modal histogram (foreground pixels and background pixels); it then enumerates the optimum threshold 
disconnecting the two classes so their combined spread (intra-class variance) is minimal. The augmentation of the original method to multi-level thresholding is referred to as the Multi Otsu method. Otsu's method is entitles after its author Nobuyuki Otsu.

An image can be described as a two-dimensional function i:e $\mathrm{f}(\mathrm{x}, \mathrm{y})$, where $\mathrm{x}$ and $\mathrm{y}$ are spatial (plane) coordinates, and the amplitude of ' $\mathrm{f}$ ' at any pair of coordinates ( $\mathrm{x}$, $\mathrm{y})$ is called the intensity or gray level of the image at that point. Each element of the array is called pixel or pel. The Pixels are stored as Integers. The integers can be of 8bit, 24-bit or 32-bit depending on the type of image. The grayscale images are 8 bit images whereas 32-bit images have an additional transparency channel. The Grayscale image consists of average of R-G-B at each pixel. It is a myth amongst people that grayscale converts colored image to image composed exclusively of shades of grey i.e. it removes color property of image. Operations are performed in some cases on each pixel of an image such as edge detection, thresholding where if there are different values of color at each pixel then it becomes more complicated. So we perform grayscale operation [1].

Histogram equalization is a technique for adjusting image intensities to increase contrast. Through this adjustment, the intensities can be better dispensed on the histogram. This lets areas of lower local contrast to yield a higher contrast. Histogram equalization achieves this by productively expanding the most persistent intensity values. The method is constructive in images with backgrounds and foregrounds that are both bright or both dark. Particularly, the method may lead to superior views of bone structure in x-ray images.

Otsu's thresholding method involves iterating through all the possible threshold values and calculating a measure of spread for the pixel levels each side of the threshold, i.e. the pixels that either fall in foreground or background. The aim is to find the threshold value where the sum of foreground and background spreads is at its minimum. In image processing, OTSU's thresholding method is used for automatic binarization level decision, based on the shape of the histogram. The algorithm assumes that the image is composed of two basic classes: Foreground and Background. It then computes an optimal threshold value that minimizes the weighted within class variances of these two classes. It is mathematically proven that minimizing the within class variance is same as maximizing the between class variance [6].

\section{Review of Similar Work}

In this section, we are discussing briefly about the earlier approaches which have been carried out in the field of image processing. The Gonzalez [1] has discussed about basics and scope of image processing. The field image processing refers to processing of digital images by means of a digital computer. Digital image is composed of a finite number of elements, each of which has a particular location and value. These elements are referred to as picture elements, image elements, pels, and pixels. Grayscale image consists of average of R-G-B at each pixel. M. Sezgin and B. Sankur (2004), "Survey over image thresholding techniques and quantitative performance 
evaluation"[3] has included the categorization of thresholding methods according to the information they are exploiting, such as histogram shape, measurement space clustering, entropy, object attributes, spatial correlation, and local gray-level surface. 40 selected thresholding methods from various categories are compared in the context of nondestructive testing applications as well as for document images. The comparison is based on the combined performance measures.

Ping-Sung Liao and Tse-Sheng Chen and Pau-Choo Chung (2001), "A Fast Algorithm for Multilevel Thresholding" [4] has proposed a faster version of Otsu's method is proposed for improving the efficiency of computation for the optimal thresholds of an image. This method can reduce down the processing time from more than one hour by the conventional Otsu's method to less than 107 seconds.

The Nobuyuki Otsu (1979), "A threshold selection method from gray-level histograms" [5] has included the optimal thresholding and global thresholding. An optimal threshold is selected by the discriminant criterion, namely, so as to maximize the separability of the resultant classes in gray levels. The procedure is very simple, utilizing only the zeroth- and the first-order cumulative moments of the gray-level histogram.

\section{Conclusion}

A method to select a threshold automatically from a gray level histogram has been derived from the viewpoint of discriminant analysis. This directly deals with the problem of evaluating the goodness of thresholds. An optimal threshold (or set of thresholds) is selected by the discriminant criterion; namely, by maximizing the discriminant measure $\mathrm{q}$ (or the measure of separability of the resultant classes in gray levels). Taking into account these points, the method suggested in this correspondence may be recommended as the most simple and standard one for automatic threshold selection that can be applied to various practical problems.

\section{Acknowledgment}

We are thankful to the Department of Computer Science \& Engineering, K. L. University, A. P. and Sinhgad Academy of Engineering, Pune (MH) for providing the support and guidance for this Research work.

\section{References}

[1] Rafael C. Gonzalez, Richard E. Woods, "Digital Image Processing", Second Edition, ISBN 0-201-18075-8.

[2] M. Sezgin and B. Sankur (2004). "Survey over image thresholding techniques and quantitative performance evaluation". Journal of Electronic Imaging 13 (1): 146-165. DOI:10.1117/1.1631315. 
[3] Ping-Sung Liao and Tse-Sheng Chen and Pau-Choo Chung (2001). "A Fast Algorithm for Multilevel Thresholding". J. Inf. Sci. Eng. 17 (5): $713-$ 727.

[4] Nobuyuki Otsu (1979). "A threshold selection method from gray-level histograms". IEEE Trans. Sys., Man., Cyber. 9 (1): 62-66. DOI:10.1109/TSMC.1979.4310076.

[5] http://en.wikipedia.org/wiki/Otsu\%27s_method

[6] http://www.labbookpages.co.uk/software/imgProc/otsuThreshold.html 
